\title{
Effects of Combined Treatment with Branched-Chain Amino Acids, Citric Acid, L-Carnitine, Coenzyme Q10, Zinc, and Various Vitamins in Tumor-Bearing Mice
}

\author{
Hiroko Awa, ${ }^{a}$ Akihiko Futamura, ${ }^{*, a}$ Takashi Higashiguchi, ${ }^{a}$ Akihiro Ito, ${ }^{a}$ Naoharu Mori, ${ }^{a}$ \\ Miyo Murai, ${ }^{a}$ Hiroshi Ohara, ${ }^{a}$ Takeshi Chihara, ${ }^{b}$ and Takaaki Kaneko ${ }^{b}$ \\ ${ }^{a}$ Department of Surgery and Palliative Medicine, School of Medicine, Fujita Health University; 1-98 \\ Dengakugakubo, Kutsukake, Toyoake, Aichi 470-1192, Japan: and ${ }^{b}$ Division of Biochemistry, Fujita Memorial \\ Nanakuri Institute, Fujita Health University; 423 Oodori, Tsu, Mie 514-1296, Japan. \\ Received August 5, 2016; accepted December 13, 2016; advance publication released online January 26, 2017
}

A functional dietary supplement (FDS) containing Coenzyme Q10, branched-chain amino acids and L-carnitine was administered to tumor-bearing mice, investigating its effects on tumor and muscle tissues. Experiment (A): B16 melanoma cells were implanted subcutaneously into the right side of the abdomen of 8- to 9-week-old C57BL/6J mice. The mice were divided into two groups: a FDS group that received oral administration of FDS $(n=10)$, and a control group that received oral administration of glucose $(n=10)$. The moribund condition was used as the endpoint, and median survival time was determined. Experiment (B): On day 21 after tumor implantation, tumors, soleus muscle, gastrocnemius muscle, and suprahyoid muscles were collected. Tumor and muscle weight and other aspects were evaluated in each group: FDS group $(n=15)$ and control group $(n=15)$. The median survival time was comparable $(21 \mathrm{~d}$ in the FDS group vs. $18 \mathrm{~d}$ in the control group, $p=0.30$ ). However, cumulative food intake was significantly higher in the FDS group than the control group $(p=0.011)$. Metastasis of melanoma to the lung was observed in the control group but not in the FDS group $(p=0.043)$. The weight of the suprahyoid muscles was significantly higher in the FDS group than in the control group $(p=0.0045)$. The weight of the tumor was significantly lower in the FDS group than in the control group $(p=0.013)$. The results possibly suggest oral administration of FDS in tumor-bearing mice enhances the maintenance of suprahyoid muscles, resulting in an extended feeding period and suppression of tumor growth and metastasis.

Key words cancer therapy; cachexia; metabolism; branched-chain amino acid; functional maintenance

Cancer cachexia is a syndrome that affects many patients with advanced cancer. It is characterized by refractory malnutrition and has significant impact on prognosis and QOL. Managing cachexia is therefore a high priority when providing palliative care. Cachexia is a complex metabolic syndrome, with loss of muscle mass being a primary symptom. Development of cachexia involves many factors and accompanies progressive functional impairment, but the pathogenesis remains unknown. ${ }^{1,2)}$

Recent studies suggest that metabolic and neuroendocrinological abnormalities in cancer cachexia are caused by inflammatory cytokines secreted by the tumor and host cells and other tumor-derived substances. It is essential for cancer patients to maintain good nutritional status for better prognosis and QOL. As such, managing cancer cachexia is a major goal for the future of cancer therapy and palliative care. Since its foundation in 2003, our laboratory has implemented a unique nutritional support system to manage cancer cachexia. $^{3-5)}$ With the nutritional support, patients with cachexia have shown reduced symptoms and improved bodily function through control of metabolic dynamics. We have developed a functional dietary supplement (FDS) for improving symptoms and function, containing Coenzyme Q10 (CoQ10), branchedchain amino acids (BCAA), L-carnitine and citric acid and reported the effectiveness of its use in clinical settings. ${ }^{6,7)}$ In this study, we have experimentally evaluated the effects of FDS on tumors and muscle tissues to corroborate the clinical findings.

\section{METHODS}

Mice C57BL/6J mice (7-week-old males) were purchased from Japan SLC Inc. (Shizuoka, Japan). After 1 to 2 weeks of acclimatization, a tumor-bearing model was established by first removing hair from the right side of the abdomen and then subcutaneously implanting B16 melanoma cells (B16F6). These mice were maintained under laboratory animal management at the Nanakuri Laboratory of Animal Models for Human Diseases, Fujita Health University. The mice were kept alone or in pairs in plastic cages with wood chip bedding and were fed normal diet MF (Oriental Yeast Co., Ltd., Tokyo, Japan) in an animal facility controlled at a temperature of $23 \pm 5^{\circ} \mathrm{C}$ with humidity at $60 \pm 5 \%$ with a 12 -h light/dark cycle.

Tumor Cells B16 melanoma cells (B16F6) have been used in tumor-bearing models and cancer cachexia models in a number of studies. ${ }^{8)}$ Because melanoma cells typically form subcutaneous tumors, the size and shape of the tumor can be easily evaluated. The highly malignant cell line B16F6 can also metastasize to the lung. ${ }^{9}$ )

B16 melanoma cells were grown in RPMI-1640 (Wako Pure Chemical Industries, Ltd., Osaka, Japan) with 10\% fetal bovine serum (Gibco, Life Technologies, Carlsbad, CA, U.S.A.) in humidified atmosphere containing $5 \% \mathrm{CO}_{2}$ at $37^{\circ} \mathrm{C}$. The medium was replaced every 2 to $3 \mathrm{~d}$, and the subcultures were obtained by harvesting using $0.25 \%$ trypsin/ethylenediaminetetraacetic acid (EDTA) (Sigma-Aldrich, St. Louis, MO, U.S.A.). 
For subcutaneous implantation, $2 \times 10^{6}$ B16 melanoma cells (B16F6) in $0.2 \mathrm{~mL}$ of the medium were prepared for each mouse and implanted into the right side of the abdomen.

Reagents The FDS sample used in this study was Inner Power $^{\circledR}$, purchased from Otsuka Pharmaceutical Co., Ltd. Each 125-g package of FDS contained $139 \mathrm{kcal}$ of energy and the following ingredients: $1.7 \mathrm{~g}$ of proteins, $0 \mathrm{~g}$ of fat, $13.5 \mathrm{~g}$ of sugars, $33 \mathrm{~g}$ of carbohydrate, $2 \mathrm{~g}$ of fiber, $90 \mathrm{mg}$ of sodium, $3 \mathrm{mg}$ of zinc, $0.3 \mathrm{mg}$ of copper, $1000 \mathrm{mg}$ of citric acid, $2500 \mathrm{mg}$ of BCAA ( $917 \mathrm{mg}$ of leucine, $833 \mathrm{mg}$ of isoleucine, $750 \mathrm{mg}$ of valine), $30 \mathrm{mg}$ of CoQ10, $50 \mathrm{mg}$ of L-carnitine, $1 \mathrm{mg}$ of vitamin $\mathrm{B} 1,3 \mathrm{mg}$ of vitamin $\mathrm{B} 2,0.9 \mathrm{mg}$ of vitamin $\mathrm{B} 6,1.5 \mu \mathrm{g}$ of vitamin B12, and $10 \mathrm{mg}$ of vitamin E. The contents of FDS were mixed in a blender into a gel consistency and administered using an oral gavage. A glucose solution was prepared $[5.56 \mathrm{~g}$ $(22.24 \mathrm{kcal})$ in $20 \mathrm{~mL}$ ] and administered to the control group.

mRNA was analyzed by real-time PCR. RNA was extracted from the gastrocnemius muscle and the tumor. mRNA for mammalian target of rapamycin (mTOR), muscle RING-finger protein-1 (MuRF1), and vascular endothelial growth factor (VEGF) were quantified.

\section{Experimental Procedures}

\section{Experiment (A)}

Male C57BL/6J mice (7 weeks old) were allowed ad libitum access to food and water and acclimatized for 1 to 2 weeks. At 8 to 9 weeks of age, B16 melanoma (B16F6) cells $\left(2 \times 10^{6}\right.$ cells in $0.2 \mathrm{~mL}$ ) were subcutaneously implanted into the right side of the abdomen (Fig. 1). The mice were divided into two groups $(n=10$ each). FDS $[0.1 \mathrm{~mL}(0.1 \mathrm{kcal}) / 10 \mathrm{~g}$ body weight $(\mathrm{BW}) / \mathrm{d}$ ] was orally administered in the FDS group, and glucose $[0.1 \mathrm{~mL}(0.1 \mathrm{kcal}) / 10 \mathrm{~g} \mathrm{BW} / \mathrm{d}]$ was orally administered in the control group. Food intake and general health status of the mice were recorded daily. The moribund condition was used as the experimental endpoint.

Experiment (B)

Tumor-bearing mice ( 8 to 9 weeks of age) were prepared as in Experiment A. The mice were divided into FDS and control groups $(n=15$ each), and their health status was recorded daily. We designed Experiment (A) and observed the condition of

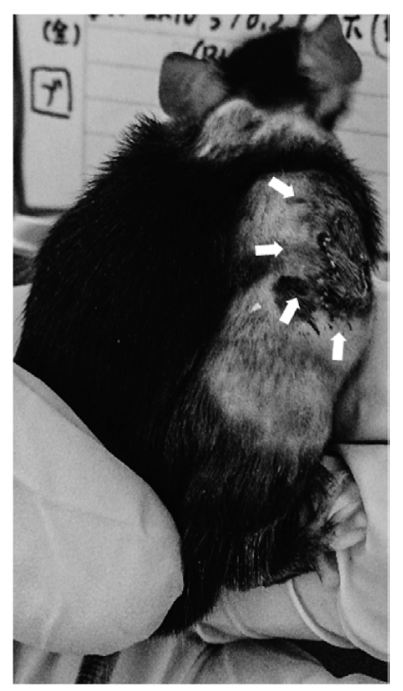

Fig. 1. Representative Photograph of Mice after Receiving B16 Melanoma Cells Implantation

B16 melanoma cells were subcutaneously implanted into the right side of the abdomen, and has been grown. the tumor-bearing mice to select a day as an endpoint. Based on the results, the endpoint was set as day 21 , which was the median survival time. On day 21 , whole blood was collected by cardiac puncture under isoflurane anesthesia. Subsequently, the tumor was removed, and the soleus and gastrocnemius muscles of both hind limbs, and suprahyoid muscles were collected. After weighing, the tissue samples were immediately frozen in liquid nitrogen and stored at $-80^{\circ} \mathrm{C}$ for protein and mRNA measurements to be conducted at a later time.

Protein contents were determined as follows: A portion of the frozen gastrocnemius muscle stored at $-80^{\circ} \mathrm{C}$ was excised and weighed. The muscle tissue was homogenized in 10 volumes of radioimmunoprecipitation assay (RIPA) buffer (Pierce Biotechnology, Rockford, IL, U.S.A.) containing 1\% protease inhibitor cocktail (Nacalai Tesque, Inc., Kyoto, Japan). The homogenate was cooling centrifuge at $13000 \times \boldsymbol{g}$ for $10 \mathrm{~min}$, and the supernatant was used for protein assay using BCA Protein Assay Kit (Pierce Biotechnology).

MuRF-1, mTOR, and VEGF mRNA levels were determined as follows: Total RNA was extracted from the tumor tissue and gastrocnemius muscle stored at $-80^{\circ} \mathrm{C}$ using High Pure RNA Tissue Kit (Roche Diagnostics GmbH, Mannheim, Germany). cDNA was synthesized using Transcriptor Universal cDNA Master Kit (Roche Diagnostics $\mathrm{GmbH}$ ) and quantified by real-time PCR using TaqMan probes. Relative mRNA expression levels were calculated by normalizing to an internal control, eukaryotic translation elongation factor 2 (eEF-2) mRNA.

Statistical Analysis Statistical analysis was performed using $^{\circledR}$ Pro 11.2.1 statistical analysis software (SAS Institute. Inc., NC, U.S.A.). To evaluate incidence of lung metastasis, difference between the FDS and control groups was determined using Fisher's exact test. For other measurements, difference between the two groups was determined using the Wilcoxon rank-sum test. All contentious variables were expressed as median and interquartile range (IQR). Survival rate was estimated using the Kaplan-Meier method with Log-rank test. The change in food intake was evaluated using two-way repeated measure ANOVA by SPSS 21 software (SPSS Inc., Chicago, IL, U.S.A.). In all analyses, $p<0.05$ was considered statistically significant.

Ethical Considerations The care and use of the animals was according to the Regulations for the Management of Laboratory Animals at Fujita Health University, which is accredited by the Japanese Association of Laboratory Animal Facilities of Public and Private Universities (JALAP). The experimental protocols were approved by the Institutional Animal Care and Use Committee of Fujita Health University (Permit No. S0141).

\section{RESULTS}

\section{Experiment (A)}

Survival Rate: The median survival time (MST) was $21 \mathrm{~d}$ (IQR: $15-27 \mathrm{~d}$ ) in the FDS group, and $18 \mathrm{~d}$ (IQR: 8-25d) in the control group ( $p=0.30)$ (Fig. 2).

Cumulative Food Intake: Food intake was analyzed for the first $9 \mathrm{~d}$ when all 10 animals were alive in both FDS and control groups. The change in food intake had significant difference between the groups (from 5.62 to $25.42 \mathrm{mg}$ in the FDS group and from 5.44 to $22.01 \mathrm{mg}$ in the control group) 


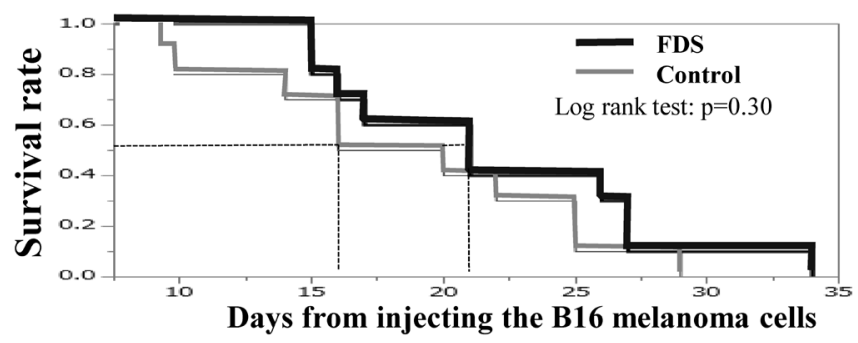

Fig. 2. Survival Rate in Tumor-Bearing Mice

The number of survival time after implanting the B16 melanoma cell to mice was measured. The median survival time (MST) was $21 \mathrm{~d}$ (IQR: 15-27d) in the FDS group, and 18d (IQR: $8-25 \mathrm{~d}$ ) in the control group.

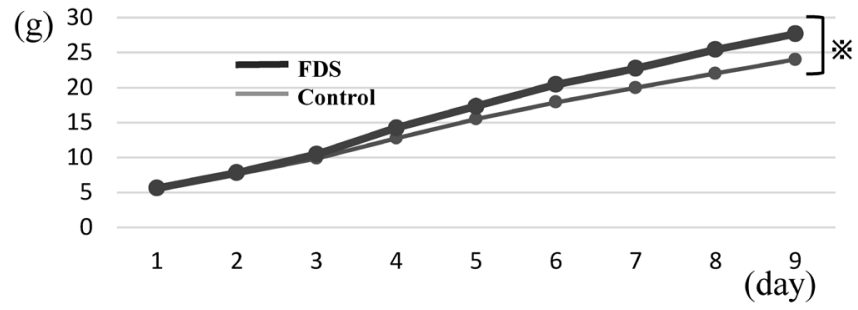

Fig. 3. Cumulative Food Intake

Food intake was analyzed for the first $9 \mathrm{~d}$ when all 10 animals were alive in both FDS and control groups. The change in food intake had significant difference between the groups $(* p=0.011)$.

\section{(Fig. 3).}

Lung Metastasis: We performed dissection of the lungs. Lung metastasis was not present macroscopically in the 10 mice in the FDS group, while lung metastasis was present macroscopically in 4 out of 10 mice in the control group $(p=0.043)$.

\section{Experiment (B)}

Loss of Body Weight: Changes in body weight from before tumor implantation to day 21 (after tumor removal) were comparable between the FDS groups [2.1\% (IQR: $-0.48-6.3)]$ and control groups [2.4\% (IQR: $-0.72-8.83)](p=0.9)$.

Muscle Tissues: Gastrocnemius and soleus muscles of both hind limbs, and suprahyoid muscles were collected, and the tissue weight was compared between the FDS and control groups. The median weight of suprahyoid muscles was $0.021 \mathrm{~g}$ (IQR: $0.018-0.024$ ) in the FDS group and $0.017 \mathrm{~g}$ (IQR: 0.017-0.020) in the control group, indicating that the FDS group maintained significantly more muscle mass $(p=0.0045)$ (Table 1). There was no significant difference in the weight of gastrocnemius and soleus muscles, and protein contents of the gastrocnemius muscle between the two groups. Relative expression levels of mTOR and MuRF1 in left gastrocnemius muscle were determined. The median mTOR expression was 0.96 (IQR: 0.89-1.06) in the FDS group and 0.94 (IQR: 0.88-1.12) in the control group. The median MuRF1 expression was 0.93 (IQR: 0.79-0.98) in the FDS group and 1.01 (IQR: 0.91-1.11) in the control group. Although expression levels of mTOR and MuRF1 were not significantly different between the two groups, the median values were higher in the FDS group, indicating a trend for better muscle growth and less muscle atrophy in the FDS group (Table 2).

Tumor Weight: The median tumor weight was significantly lower in the FDS group [4.50g (IQR: 3.96-5.44)] compared to the control group [6.08g (IQR: 5.12-6.76)] $(p=0.013)$

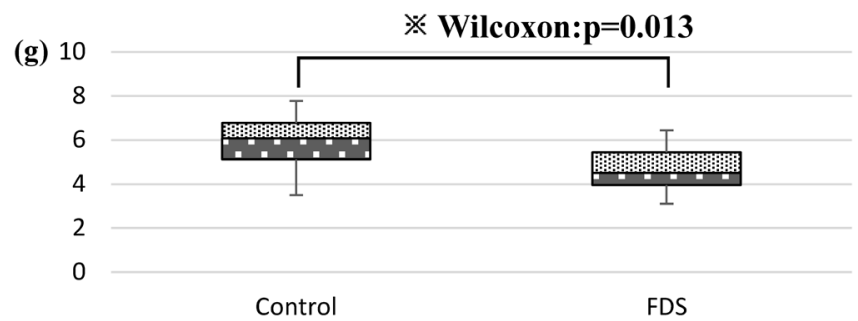

Fig. 4. Tumor Weight in Tumor-Bearing Mice

On day 21 after tumor was implanted to mice, whole blood was collected by cardiac puncture under isoflurane anesthesia, subsequently the tumor was removed. The median tumor weight was significantly lower in the FDS group $[4.50 \mathrm{~g}$ (IQR: $3.96-5.44)$ ] compared to the control group [6.08g (IQR: $5.12-6.76)]\left({ }^{*} p=0.013\right)$.

Table 1. Weight of Muscle Tissue in Tumor-Bearing Mice

\begin{tabular}{lccc}
\hline \hline & \multicolumn{3}{c}{ Weight $(\mathrm{g})$} \\
\cline { 2 - 4 } & $\begin{array}{c}\text { Gastrocnemius } \\
\text { muscles }\end{array}$ & $\begin{array}{c}\text { Soleus } \\
\text { muscles }\end{array}$ & $\begin{array}{c}\text { Suprahyoid } \\
\text { muscles }\end{array}$ \\
\hline \multirow{2}{*}{ FDS } & 0.23 & 0.015 & 0.021 \\
Control & $(0.22-0.24)$ & $(0.014-0.017)$ & $(0.018-0.024)$ \\
$p$ Value & 0.22 & 0.014 & 0.017 \\
\hline & $0.21-0.23)$ & $(0.014-0.016)$ & $(0.017-0.02)$ \\
& & 0.24 & $0.0045^{*}$ \\
\hline
\end{tabular}

Gastrocnemius and soleus muscles of both hind limbs, and suprahyoid muscles were collected, and the tissue weight was compared between the FDS and control groups $\left({ }^{*} p=0.0045\right)$.

Table 2. Relative Expression Levels of mTOR and MuRF1 and Protein Contents in Left Gastrocnemius Muscle

\begin{tabular}{lccc}
\hline \hline & $\begin{array}{c}\text { Protein contents } \\
(\mu \mathrm{g} / \mathrm{g})\end{array}$ & mTOR & MuRF1 \\
\cline { 2 - 4 } & \multicolumn{3}{c}{ Gastrocnemius muscles } \\
\hline \multirow{2}{*}{ FDS } & 66.8 & 0.96 & 0.93 \\
& $(63.6-68.49)$ & $(0.89-1.06)$ & $(0.79-0.98)$ \\
Control & 65.16 & 0.94 & 1.01 \\
p Value & $(62.32-66.84)$ & $(0.88-1.22)$ & $(0.91-1.11)$ \\
& 0.28 & 0.65 & 0.13 \\
\hline
\end{tabular}

The median mTOR expression was 0.96 (IQR: 0.89-1.06) in the FDS group and 0.94 (IQR: 0.88-1.12) in the control group. The median MuRF1 expression was 0.93 (IQR: $0.79-0.98$ ) in the FDS group and 1.01 (IQR: 0.91-1.11) in the control group.

(Fig. 4). When the ratio of the tumor weight to the net body weight (weight after tumor removal) was compared, the median value was significantly lower in the FDS group [21.1\% (IQR: 17.39-24.95)] than in the control group [27.1\% (IQR: 23.24-31.05)] ( $p=0.016)$ (Fig. 5).

Relative mTOR and VEGF Expression Levels in the Tumor Tissue: The median value of relative mTOR expression within the tumor tissue showed no significant difference between the FDS group [0.96 (IQR: 0.84-1.05) $(n=12)]$ and the control group [0.98 (IQR: 0.86-1.09) $(n=12)]$ $(p=0.82)$. The median value of relative VEGF expression didn't show significant difference between the FDS group [0.70 (IQR: $0.59-0.80)(n=12)]$ and the control group [0.67 (IQR: 0.64-0.90) $(n=12)](p=0.79)($ Table 3). 


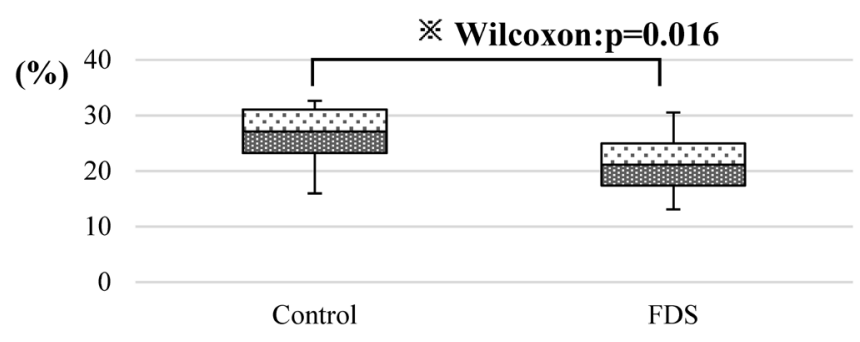

Fig. 5. The Ratio of Tumor Weights to Body Weights

The ratio of the tumor weight to the net body weight (weight after tumor removal) was compared. The median value was significantly lower in the FDS group [21.1\% (IQR: 17.39-24.95)] than in the control group [27.1\% (IQR: 23.24-31.05)] $(* p=0.016)$.

Table 3. Relative mTOR and VEGF Expression Levels in the Tumor Tissue

\begin{tabular}{lcc}
\hline \hline & mTOR & VEGF \\
\cline { 2 - 3 } & \multicolumn{2}{c}{ Tumor tissue } \\
\hline \multirow{2}{*}{ FDS } & 0.96 & 0.70 \\
& $(0.84-1.05)$ & $(0.59-0.80)$ \\
Control & 0.95 & 0.67 \\
$p$ Value & $(0.86-1.09)$ & $(0.64-0.90)$ \\
\hline
\end{tabular}

Median (IQR)

The median value of relative mTOR expression within the tumor tissue showed no significant difference between the FDS group [0.96 (IQR: $0.84-1.05)(n=12)$ and the control group [0.98 (IQR: 0.86-1.09) $(n=12)](p=0.82)$. The median value of relative VEGF expression didn't show significant difference between the FDS group [0.70 (IQR: $0.59-0.80)(n=12)]$ and the control group [0.67 (IQR: 0.64-0.90) $(n=12)](p=0.79)$.

\section{DISCUSSION}

In our ongoing effort, we have developed a dietary supplement for improving symptoms and function, named "CoQ10+BCAA+L-carnitine (FDS)," and reported its effectiveness in clinical cases of cachexia. To our knowledge, this study is the first to evaluate the effects of FDS on tumors and muscle tissues, focusing on mechanistic aspects. The results possibly suggest oral administration of FDS in tumor-bearing mice enhances the maintenance of suprahyoid muscles, resulting in an extended feeding period and suppression of tumor growth and metastasis.

The most important issue in this study, cachexia-associated loss of suprahyoid muscles was significantly reduced in the FDS group. To date, there is no report on the effect of cancer cachexia on muscles involved in mastication, including suprahyoid muscles. Our study revealed that cancer cachexia may affect mastication muscles before affecting hind limb muscles, and that FDS administration could ameliorate the sarcopenia. In cancer cachexia-associated anorexia, ghrelin has drawn much attention because of its role in the regulation of gastrointestinal activity and energy consumption. ${ }^{10)}$ Our findings indicate that impaired mastication should also be considered as a cause of anorexia.

In addition we have evaluated several muscles: suprahyoid muscles, which are important in deglutition; the fast-twitch gastrocnemius muscle; and the slow-twitch soleus muscle.

We also investigated mTOR, which has a critical role in tumor growth. ${ }^{11)}$ However, since there is no difference in the mTOR muscle tissue as well as tumor tissue, FDS may not affect the mTOR. In the weight of the muscular tissue, the possibility that FDS affected MuRF1, an amyotrophycausing gene, ${ }^{12)}$ was shown. On the significant difference in tumor weight, an unknown mechanism different from mTOR pathway might participate in suppression of tumor growth. For example, the antitumor effect of these nutrients such as L-carnitine, citrate, CoQ10 and zinc may also be considered.

There was no significant difference in the number of days of survival of tumor-bearing mice between the FDS group and the control (glucose) group. However, the most important aspect of this study was a difference in the median values, reflecting the fact that more animals in the control group experienced sudden death early in the study compared to the FDS group. The reason for this could be that significantly more mice had lung metastases in the control group, affecting the median value of survival time.

There are different opinions about the timing of sample collection from mice. Some studies set the endpoint as the time when the melanoma reached a certain size. ${ }^{13,14)}$ Others set the endpoint as a certain day (from day 6 to 28) after melanoma cells were implanted, though the basis for selecting the day was not provided. ${ }^{15-18)}$ In the beginning of our study, we considered measuring the tumor volume over time, but opted not to do so because of the inaccuracy due to the irregular shape of the tumor formed by the melanoma cells used in the study. We then designed Experiment (A) and observed the condition of the tumor-bearing mice to select a day as an endpoint. Based on the results, the endpoint was set as day 21, which was the median survival time. With this endpoint, we may have evaluated an early cachexic state. In the future, a later endpoint should be set to investigate an advanced cachexic state.

FDS also contains L-carnitine, citric acid, CoQ10, and zinc, each of which is expected to have antitumor and anti-inflammatory effects.

Huang et al. ${ }^{19)}$ reported that L-carnitine therapy reduced ATP production and increased p21 mRNA and protein in hepatoma cells (HepG2, SMMC-7721). Further, L-carnitine acted as an intrinsic inhibitor of histone deacetylase (HDAC). Based on these findings, L-carnitine therapy is expected to have antitumor effects.

Citrate inhibits phosphofructokinase 2 (PFK2) in cancer cells. Overexpression of the nuclear isoform of PFK2 facilitates cancer cell growth by modulating central cell cycle regulatory factors. Conversely, selective inhibition of PFK2 suppresses cell growth. Further, citrate slows the activity of pyruvate dehydrogenase $(\mathrm{PDH})$ at the entry into the tricarboxylic acid (TCA) cycle and inhibits the exit by interacting with succinate dehydrogenase. Citrate also indirectly inhibits $\beta$-oxidation. Thus, citrate can fine-tune the metabolic flow and ATP production by regulating strategic enzymes at the entry and exit of glycolysis, TCA cycle, gluconeogenesis, and fatty acid synthesis, all of which leads to suppression of cancer cells. $^{20)}$

Effect of CoQ10 against hepatocellular carcinoma was reported by Fouad et al. ${ }^{21)}$ CoQ10 significantly reduced peroxidation of lipids and prevented the loss of reduced glutathione (GSH) and superoxide dismutase activity (SOD). It also reduced the increase of tumor necrosis factor- $\alpha$ (TNF- $\alpha$ ) and nitric oxide (NO) in the liver of the rats with hepatocellular carcinoma. Histopathology of the liver revealed that dysplastic 
changes were ameliorated by CoQ10.

Antioxidant effect of zinc was reviewed by Prasad. ${ }^{22)}$ In a human model of zinc deficiency, even mild zinc deficiency caused an increase in interleukin (IL)- $1 \beta$ production in monocytes ex vivo. Wong et al. ${ }^{23)}$ reported that zinc deficiency caused abnormal activation of immune cells and dysregulation of IL-6. Taken together, these findings suggest that zinc deficiency itself can activate macrophages, induce proinflammatory cytokines, and increase oxidative stress.

There is a concern that one of the BCAA components in FDS, leucine, may facilitate tumor growth. Liu et al. ${ }^{24)}$ reported that leucine supplementation enhanced pancreatic cancer growth in both lean and overweight mice. On the other hand, Diaz-Meco ${ }^{25}$ ) demonstrated that melanoma cells were highly sensitive to leucine deprivation, which caused inhibition of autophagy.

Reference Intake for Japanese set by the Ministry of Health, Labour and Welfare is $39 \mathrm{mg}$ of leucine, $20 \mathrm{mg}$ of isoleucine, and $26 \mathrm{mg}$ of valine per $1 \mathrm{~kg}$ of body weight. ${ }^{26)}$ These amounts roughly correspond to 2 packs of Inner Power ${ }^{\circledR}$ per day for a $60 \mathrm{~kg}$ human. The BCAA dose used in our study was in accordance with the recommended volume for mice, $0.1 \mathrm{~mL} / 10 \mathrm{~g}$ $\mathrm{BW},{ }^{27)}$ which contained $0.7336 \mathrm{mg}$ of leucine, $0.6664 \mathrm{mg}$ of isoleucine, and $0.6 \mathrm{mg}$ of valine per $10 \mathrm{~g}$ of body weight. In animal studies, the equivalent doses of test substances are often tens of times higher than standard doses in humans. The dose we used in the current study was relatively low (equivalent to 2 to 3 times the standard dose for humans). While effect of BCAA alone on protein synthesis in cancer patients has been evaluated, ${ }^{28)}$ data are scarce for complex mixtures such as FDS. We therefore used the original composition of Inner Power ${ }^{\circledR}$ in this study.

In future study, the dose load should be optimized by extracting individual components of FDS and creating various combinations of the components.

The results suggest the possibility that oral administration of FDS in tumor-bearing mice enhances the maintenance of suprahyoid muscles, resulting in an extended feeding period and suppression of tumor growth and metastasis.

Acknowledgments We deeply thank Drs. Mie Shimizu, Ayako Nakagawa, Norimasa Tsuzuki, Ayako Miyoshi and Yoshihiro Uekuzu (Department of Surgery and Palliative Medicine, Fujita Health University School of Medicine) for continued guidance. We sincerely thank Assoc. Prof. Masahiro Ohsawa (Department of Neuropharmacology, Nagoya City University) for his guidance in research principles and initiative, and for providing B16 melanoma cells, which were essential for this study. We also thank Dr. Kan Shimpo (Division of Biochemistry, Fujita Memorial Nanakuri Institute, Fujita Health University) for providing the research environment and guidance. We deeply thank Dr. Hiroshi Takahashi (Director, Division of Medical Statistics Fujita Health University) for his assistance in statistical analysis. We sincerely thank Shizuko Nagao, Ph.D. (Education and Research Center of Animal Models for Human Diseases, Fujita Health University) for her guidance in cell cultures and handling animals.

Conflict of Interest The authors declare no conflict of interest.

\section{REFERENCES}

1) August DA. Nutritional Care of Cancer patients. Surgery. Basic Science and Clinical Evidence. (Norton JA, Bollinger PR, Chang AE, Lowry SF, Mulvihill SJ, Pass HI, Thompson RW eds.) SpringerVerlag, New York, pp. 1841-1862 (2000).

2) Tisdale MJ. Pathogenesis of cancer cachexia. J. Support. Oncol., 1, 159-168 (2003).

3) Higashiguchi $T$, Ito $A$, Iida $T$, Murai M. Fluid therapy in terminal cancer patients. Japan. Med. Assoc. J., 132, 61-64 (2004).

4) Higashiguchi T. Nutritional management for the elderly and terminally ill patients. Byouin, 65, 146-151 (2006).

5) Higashiguchi T. The roles of a nutrition support team. Nippon Geka Gakkai Zasshi, 105, 206-212 (2004).

6) Higashiguchi T, Futamura A, Ito A. Effect of a complementary nutrition diet for improving clinical condition and function in terminal cancer patients: A controlled clinical trial. Jpn. J. Surg. Metab. Nutr., 44, 157-169 (2010).

7) Higashiguchi $T$, Futamura $A$, Ito A, Yaga $S$, Ohkawa $T$, Ohkawa H. A clinical study of the immediate and delayed effects of new dietary supplements on exercise-related fatigue and the inhibition of delayed-onset muscle soreness. Ann. Public Health Res., 1, 1010 (2014).

8) Das SK, Eder S, Schauer S, Diwoky C, Temmel H, Guertl B, Gorkiewicz G, Tamilarasan KP, Kumari P, Trauner M, Zimmermann R, Vesely P, Haemmerle G, Zechner R, Hoefler G. Adipose triglyceride lipase contributes to cancer-associated cachexia. Science, 333, 233-238 (2011).

9) Peinado $\mathrm{H}$, Alečković $M$, Lavotshkin S, Matei I, Costa-Silva B, Moreno-Bueno G, Hergueta-Redondo M, Williams C, GarcíaSantos G, Ghajar C, Nitadori-Hoshino A, Hoffman C, Badal K, Garcia BA, Callahan MK, Yuan J, Martins VR, Skog J, Kaplan RN, Brady MS, Wolchok JD, Chapman PB, Kang Y, Bromberg J, Lyden D. Melanoma exosomes educate bone marrow progenitor cells toward a pro-metastatic phenotype through MET. Nat. Med., 18, 883-891 (2012)

10) Fujitsuka N, Uezono Y. Rikkunshito, a ghrelin potentiator, ameliorates anorexia-cachexia syndrome. Front Pharmacol., 5, 271 (2014).

11) Nishitani S, Horie M, Ishizaki S, Yano H. Branched-chain amino acid suppresses hepatocellular cancer stem cells through the activation of mammalian target of rapamycin. PLoS ONE, 8, e82346 (2013).

12) Bodine SC, Baehr LM. Skeletal muscle atrophy and the E3 ubiquitin ligases MuRF1 and MAFbx/atrogin-1. Am. J. Physiol. Endocrinol. Metab., 307, E469-E484 (2014).

13) Moskalenko M, Pan M, Fu Y, de Moll EH, Hashimoto D, Mortha A, Leboeuf M, Jayaraman P, Bernardo S, Sikora AG, Wolchok J, Bhardwaj N, Merad M, Saenger Y. Requirement for innate immunity and CD90 ${ }^{+}$NK1.1 lymphocytes to treat established melanoma with chemo-immunotherapy. Cancer Immunol. Res., 3, 296-304 (2015).

14) Kayaga J, Souberbielle BE, Sheikh N, Morrow WJW, Scott-Taylor T, Vile R, Chong H, Dalgleish AG. Anti-tumour activity against B16-F10 melanoma with a GM-CSF secreting allogeneic tumour cell vaccine. Gene Ther., 6, 1475-1481 (1999).

15) Bruns H, Bessell C, Varela JC, Haupt C, Fang J, Pasemann S, Mackensen A, Oelke M, Schneck JP, Schütz C. CD47 enhances in vivo functionality of artificial antigen-presenting cells. Clin. Cancer Res., 21, 2075-2083 (2015).

16) Cantor JM, Rose DM, Slepak M, Ginsberg MH. Fine-tuning tumor immunity with integrin trans-regulation. Cancer Immunol. Res., $\mathbf{3}$, 661-667 (2015).

17) Das SK, Eder S, Schauer S, Diwoky C, Temmel H, Guertl B, Gorkiewicz G, Tamilarasan KP, Kumari P, Trauner M, Zimmermann R, Vesely P, Haemmerle G, Zechner R, Hoefler G. Adipose triglyceride lipase contributes to cancer-associated cachexia. Sci- 
ence, 333, 233-238 (2011)

18) Li H, Gupta S, Du WW, Yang BB. MicroRNA-17 inhibits tumor growth by stimulating T-cell mediated host immune response. Oncoscience, 1, 531-539 (2014).

19) Huang H, Liu N, Guo H, Liao S, Li X, Yang C, Liu S, Song W, Liu C, Guan L, Li B, Xu L, Zhang C, Wang X, Dou QP, Liu J. L-Carnitine is an endogenous HDAC inhibitor selectively inhibiting cancer cell growth in vivo and in vitro. PLoS ONE, 7, e49062 (2012).

20) Icard P, Poulain L, Lincet H. Understanding the central role of citrate in the metabolism of cancer cells. Biochim. Biophys. Acta, 1825, 111-116 (2012).

21) Fouad AA, Al-Mulhim AS, Jresat I. Therapeutic effect of coenzyme Q10 against experimentally-induced hepatocellular carcinoma in rats. Environ. Toxicol. Pharmacol., 35, 100-108 (2013).

22) Prasad AS. Zinc: An antioxidant and anti-inflammatory agent: Role of zinc in degenerative disorders of aging. J. Trace Elem. Med. Biol., 28, 364-371 (2014).

23) Wong CP, Rinaldi NA, Ho E. Zinc deficiency enhanced inflamma- tory response by increasing immune cell activation and inducing IL6 promoter demethylation. Mol. Mutr. Food Res., 59, 991-999 (2015)

24) Liu KA, Lashinger LM, Rasmussen AJ, Hursting SD. Leucine supplementation differentially enhances pancreatic cancer growth in lean and overweight mice. Cancer Metab., 2, 6 (2014).

25) Diaz-Meco MT. Targeting leucine addiction and autophagy in melanoma. Pigment Cell Melanoma Res., 24, 588-589 (2011).

26) $\mathrm{WHO} / \mathrm{FAO} / \mathrm{UNU}$. Protein and amino acid requirements in human nutrition. WHO Technical Report Series 935, WHO, Geneva. (2007).

27) Diehl KH, Hull R, Morton D, Pfister R, Rabemampianina Y, Smith D, Vidal JM, van de Vorstenbosch C. A good practice guide to the administration of substances and removal of blood, including routes and volumes. J. Appl. Toxicol., 21, 15-23 (2001).

28) Eley HL, Russell ST, Tisdale MJ. Effect of branched-chain amino acids on muscle atrophy in cancer cachexia. Biochem. J., 407, $113-120$ (2007). 